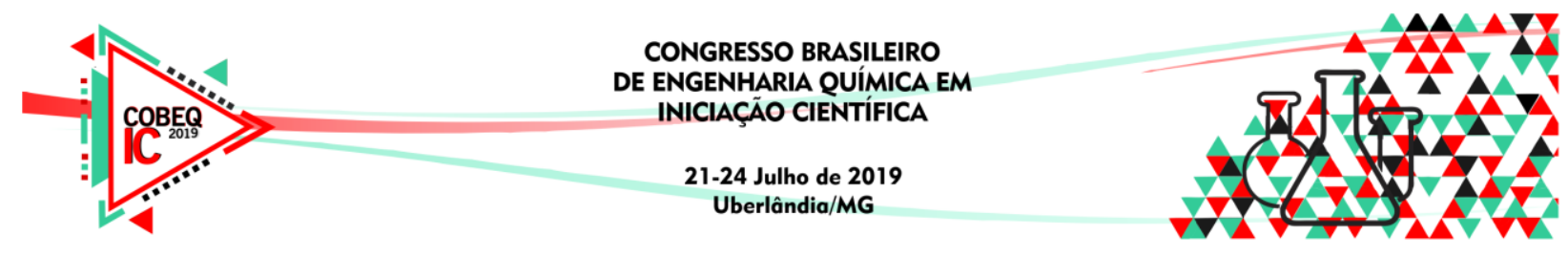

\title{
ESTUDO CDF DA REFORMA A VAPOR DO ETANOL, VIA REAÇÕES CATALÍTICAS
}

\author{
A. L. G. EURIDES ${ }^{1}$, I. M. ALVES ${ }^{1}$, L. S. FERNANDES ${ }^{1}$, L. H. ANDRADE ${ }^{1}$, C. H. G. \\ BRITO $^{2}$ \\ ${ }^{1}$ Pontifícia Universidade Católica de Minas Gerais, Departamento de Engenharia Química \\ ${ }^{2}$ Pontifícia Universidade Católica de Minas Gerais, Departamento de Engenharia Mecânica \\ E-mail para contato: analuiza.eurides@ hotmail.com
}

\begin{abstract}
RESUMO - A demasiada utilização de combustíveis fósseis acarretou em diversos problemas ambientais, que impactam diretamente a vida dos seres vivos, implicando na criação e adoção de legislações rígidas. Faz-se necessário estudos que visem a substituição daqueles por fontes limpas e renováveis, que não afetem a eficiência e aplicabilidade dos processos. Assim, o hidrogênio tornou-se uma alternativa interessante por poder ser obtido de fontes renováveis, inesgotáveis e não poluentes. Assim, esse trabalho propõe a simulação da reforma a vapor de etanol sob níquel, objetivando a formação de hidrogênio. O software Ansys Fluent foi utilizado, e variáveis temperatura e proporção vapor/etanol foram avaliadas. Pode-se perceber que o aumento da razão molar vapor/etanol desfavorece a ocorrência da reação que ocorre em maior extensão.
\end{abstract}

\section{INTRODUÇÃO}

O aumento descontrolado de fontes poluidoras tem contribuído para a redução da qualidade do ar e as fontes veiculares têm um papel significativo nesse efeito, especialmente nos grandes centros urbanos. As emissões veiculares carregam em sua composição várias substâncias que podem causar efeitos negativos na saúde humana, animal e vegetal, como deterioração do património e recursos naturais (Teixeira et al., 2016). Nesse contexto, muito se estuda sobre o desenvolvimento de novas tecnologias, que visem reduzir as taxas de emissões de poluentes, para cumprir com as legislações que vêm se tornando cada vez mais rigorosas, e manter, no mínimo, o padrão de eficiência dos motores atuais (Chaichan, 2018).

Pesquisas mostraram que o hidrogênio $\left(\mathrm{H}_{2}\right)$ é um combustível alternativo promissor, pois possui excelentes propriedades de combustão, não emite gases poluentes, e principalmente, é de origem limpa e renovável e pode ser gerado de diferentes maneiras (Chen et al., 2004). A reforma a vapor é um processo de produção de hidrogênio, altamente endotérmico, envolvendo uma reação entre um combustível hidrocarboneto e a água, com auxílio de catalisadores, resultando em uma mistura gasosa contendo dióxido de carbono, monóxido de carbono, metano, hidrogênio e água (Teixeira et al., 2016). O combustível que vem se destacando nesse cenário é o etanol, principalmente por ser obtido a partir de fontes renováveis e através de processos menos agressivos ao meio ambiente (Maia et al., 2007). 


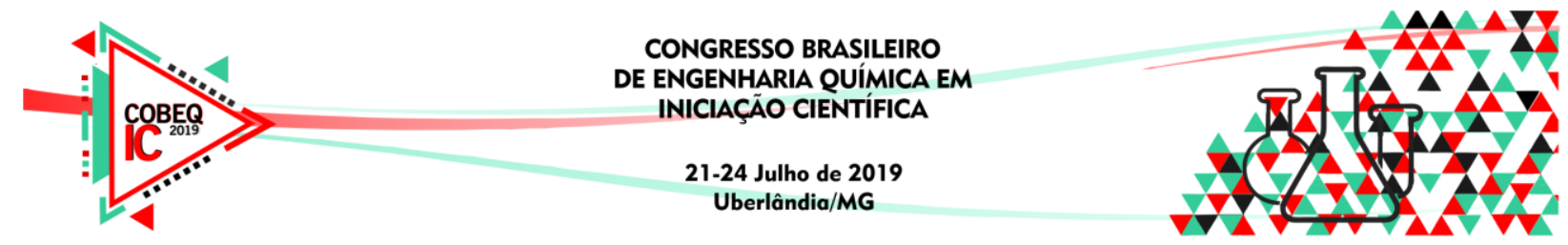

Para identificar as condições que maximizam a produção de hidrogênio, estudos que analisam os parâmetros da reforma têm recebido grande atenção entre os pesquisadores da área (Vicente et al., 2014). Segundo Sun et al. (2012), o aumento da temperatura promove um aumento na conversão do etanol e na produção de hidrogênio. Vicente et al. (2014) encontraram conversão completa de etanol a $700{ }^{\circ} \mathrm{C}$ usando níquel como catalisador. Ainda de acordo com eles, o aumento da razão molar vapor/etanol aumenta quase linearmente a conversão de etanol e a produção de $\mathrm{H}_{2}$. Para razão vapor/etanol de 10 , obteve-se $98 \%$ de conversão e $49 \%$ de rendimento, sendo o rendimento dado pela produção real de $\mathrm{H}_{2}$ relacionado com a produção se $100 \%$ de etanol fosse convertido em $\mathrm{H}_{2}$. Para otimizar o desempenho dos motores, no que diz respeito ao fluxo de gás e à transferência de calor, estudos dos gases de comportamento dinâmico através de reformadores catalíticos foram iniciados. Com o grande avanço técnico-científico com o passar dos anos, tais estudos foram explorados através de simulações computacionais, que permitem abordar fenômenos mais detalhados, principalmente os eventos que ocorrem no catalisador (Martins, 2006).

O objetivo deste trabalho foi simular a reforma a vapor do etanol sobre o níquel, usando o Ansys Fluent ® 14.5 software de simulação, incorporando algumas condições de trabalho e reações químicas envolvidas no processo. Será avaliada a influência da temperatura e da razão vapor/etanol, no que tange a formação de hidrogênio.

\section{MODELAGEM MATEMÁTICA}

A modelagem matemática do problema baseia-se na resolução das equações de conservação para os volumes fixos definidos pela discretização do domínio, utilizando o método dos volumes finitos. As equações de conservação de massa, quantidade de movimento (Navier-Stokes) e energia são descritas a seguir. Todo equacionamento apresentado foi obtido de Ansys (2013).

Equação da continuidade:

$$
\frac{\partial p}{\partial t}+\nabla \cdot(\rho \mathbf{v})=0
$$

Equação da quantidade de movimento:

$$
\frac{\partial}{\partial t}(\rho \mathbf{v})+\nabla \cdot(\rho \mathbf{v} \times \mathbf{v})=-\Delta p+\nabla \cdot(\overline{\bar{\tau}})+\rho \mathbf{g}
$$

em que $\overline{\bar{\tau}}$ é o tensor de tensão.

Equação da conservação de energia:

$$
\frac{\partial(\rho E)}{\partial t}+\nabla \cdot((\rho V+\mathrm{p}) \mathbf{v})=\nabla \cdot\left(\lambda \nabla T+\overline{\bar{\tau}} \cdot \mathbf{v}-\Sigma_{j} h_{j} J_{j}\right)+S_{h}
$$

em que h é a entalpia termodinâmica, $\lambda$ é a condutividade térmica e T é a temperatura.

Balanço de massa dos componentes: 


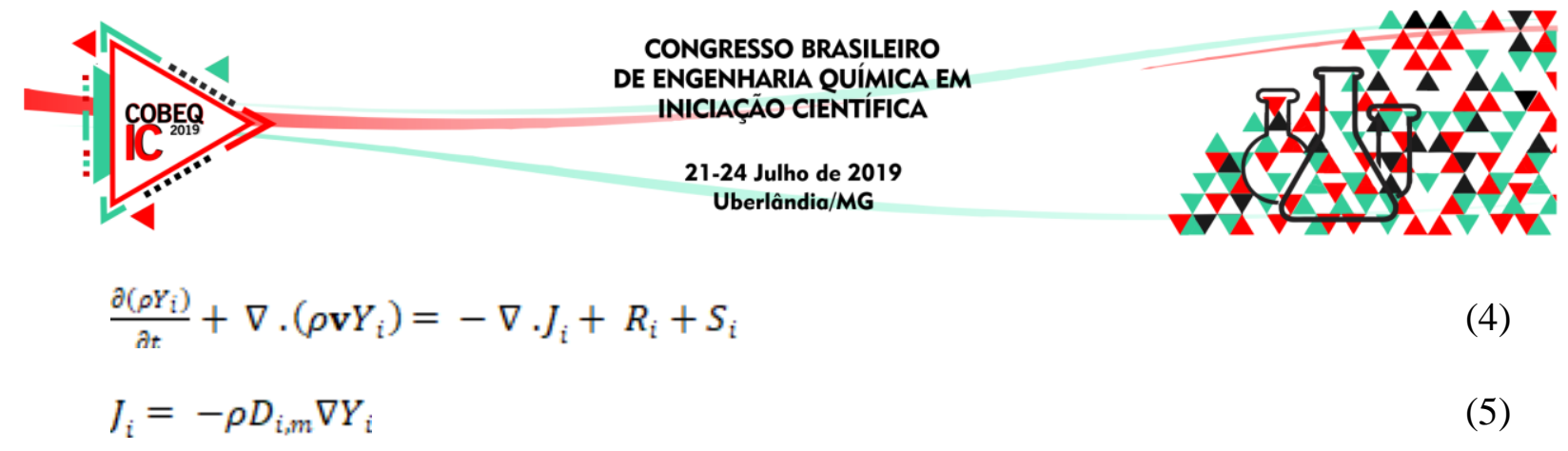

em que $\mathrm{Y}_{i}$ é a fração mássica da espécie ${ }_{i}, \mathrm{R}_{i}$ é a taxa de produção por reação química, $\mathrm{S}_{i}$ é a taxa de criação por adição da fase dispersa e $\mathrm{D}_{i, m}$ é o coeficiente de difusão da espécie ${ }_{i}$ na mistura.

\section{METODOLOGIA NUMÉRICA}

Toda simulação foi conduzida com o programa comercial Ansys Fluent ® 14.5 (2013) em computador Windows 764 bits, com 32 GB de memória RAM, equipado com processador Intel® Xeon ${ }^{\mathrm{TM}}$ CPU E5-1660 @ 3,30 Ghz de 6 núcleos.

\subsection{Elaboração da Geometria}

A fim de se realizar a reforma in situ, gerando hidrogênio no local onde ele será consumido, essa ocorreu em um conversor catalítico automotivo de um motor a diesel, constituído de vários microcanais, que servem de leito para as reações. Uma vez que todos os canais do conversor são semelhantes e simétricos, o trabalho se concentrou na simulação de um único microcanal, e a título de simplificação, elaborou-se a geometria que demandasse menos tempo e recurso de processamento. O domínio computacional construído consistiu em $1 / 8$ de um microcanal de $40 \mathrm{~mm}$ de comprimento e arestas de $0,450 \mathrm{~mm}, 0,450 \mathrm{~mm}$ e $0,636 \mathrm{~mm}$ de arestas, conforme representado na Figura1.

Figura 1 - Representação da geometria simulada

(a) Representação da divisão feita no canal. (b) Microcanal utilizado já discretizado.
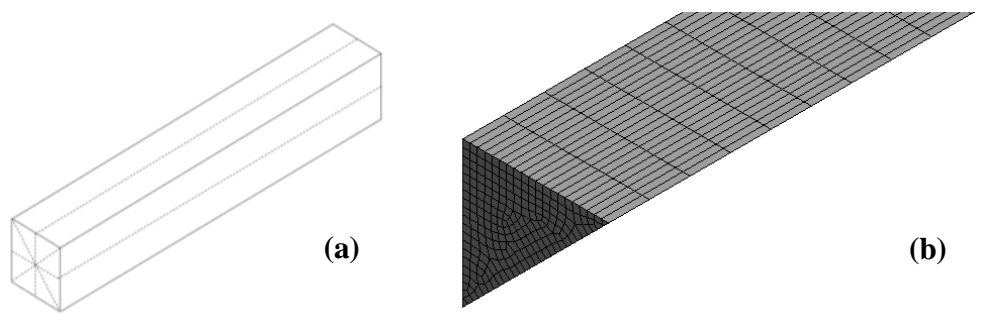

\subsection{Geração da malha}

Criou-se a malha do tipo estruturada, a fim de se garantir que esta fosse constituída exclusivamente de elementos hexaédricos. Utilizou-se o Sweep Method, o qual gera malha quadrilátera em uma superfície inicial, que é extrudida ao longo de todo o domínio até a superfície final. Na região de entrada do microcanal aplicou-se um refinamento maior, por esta área ser considerada crítica na modelagem do problema, devido ao processamento das reações químicas. Ao final da discretização, obteve-se uma malha composta de 210.000 células, 662.210 face e 243.243 nós.

\subsection{Condições de contorno e entrada}




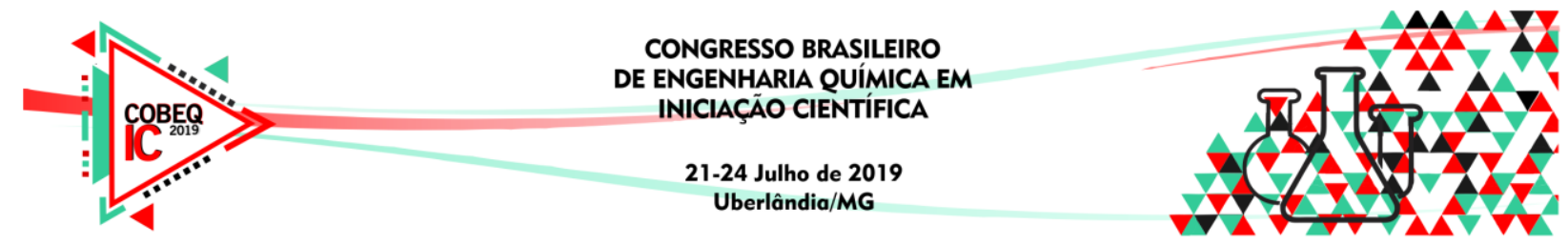

As hipóteses assumidas para a simulação foram: escoamento em regime permanente, incompressível e laminar; comportamento ideal dos gases; e radiação desprezada. A Figura 2 mostra a representação esquemáticas das condições de contorno para o microcanal.

Na modelagem química ao longo do escoamento considerou-se: reações em fase gasosa e heterogênea, sendo as reações heterogêneas fonte de calor; difusão na entrada; energia gerada na difusão; difusão térmica; e que as reações são limitadas pelo equilíbrio. Quanto às reações químicas envolvidas no processo, será utilizado um modelo microcinético, baseado na teoria do funcional da densidade (DFT, do inglês Sendity Functional Theory) proposto por Catapan (2012). Foram selecionadas as reações mais significativas para o processo de reforma, segundo o autor.

Figura 2 - Condições de contorno adotadas

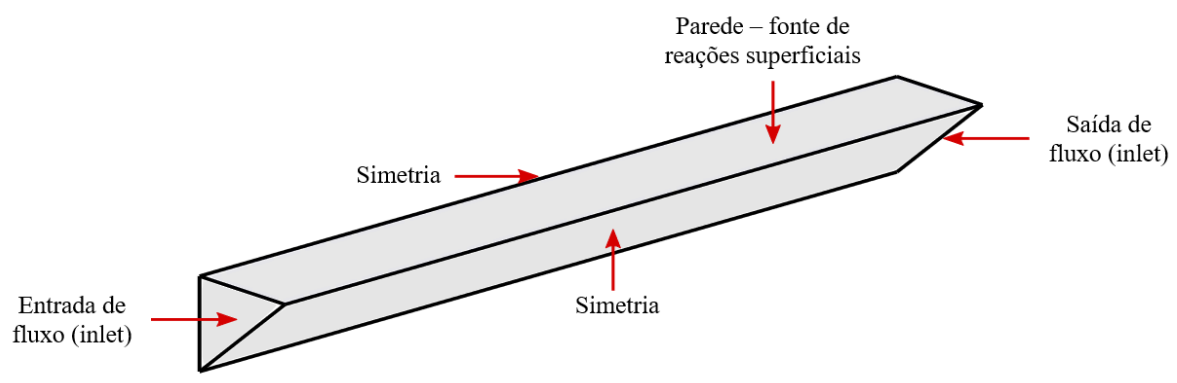

A simulação foi realizada na razão vapor/etanol de 6:1 e a uma temperatura de 852,35 K. A velocidade dos gases estipulada na entrada foi de $0,01 \mathrm{~m} / \mathrm{s}$. O número máximo de iterações assumidas foi 1.500 .

\section{RESULTADOS E DISCUSSÃO}

As Figuras 3 e 4 foram obtidas como resultado da simulação, para razão vapor/etanol de 6:1, na temperatura de 852,35 K. Na Figura 3 observa-se o perfil da taxa da reação de formação de hidrogênio, a partir do etanol, formando também o acetaldeído, Equação 6, reação mais significativa para o processo de reforma simulado. A ocorrência de tal reação em proporções maiores, ao comparada com as outras reações da reforma, evidencia que a escolha operacional da simulação não foi interessante, visando a produção de hidrogênio. Isto porque, a reação de reforma tem caminhos preferenciais para ocorrência, que dependem do catalisador utilizado e das condições operacionais (MA et al., 2016; VICENTE et al., 2014).

$$
\mathrm{CH}_{3} \mathrm{CH}_{2} \mathrm{OH} \rightarrow \mathrm{CH}_{3} \mathrm{CHO}+\mathrm{H}_{2}
$$

Observa-se através da Figura 3, que a reação citada ocorre com maior intensidade na região de entrada do canal, com uma taxa máxima de 2455,3 $\mathrm{mol} / \mathrm{m}^{3}$.s. Na Figura 4 está representado o perfil de temperatura no canal, e é possível observar uma zona de menor temperatura coincidente com a zona onde há maior ocorrência da reação química, devido ao seu caráter endotérmico. $\mathrm{O}$ aumento da temperatura volta a aumentar no canal, pois as paredes continuam numa temperatura elevada, aquecendo o gás remanescente. 


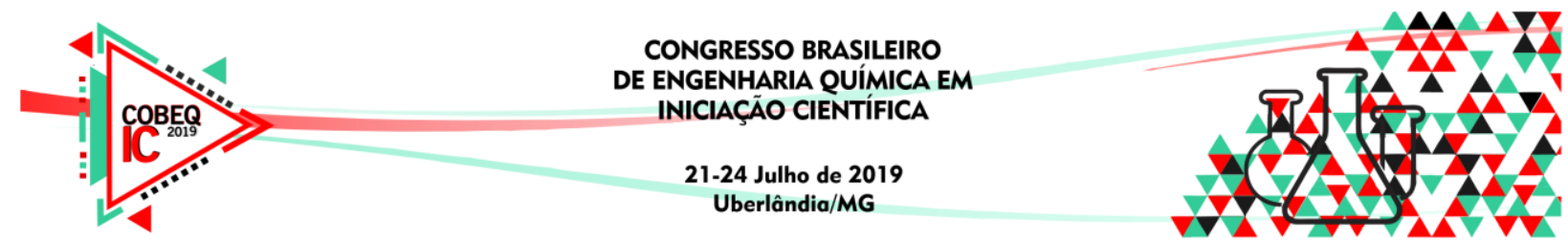

Figura 3 - Taxa da reação da formação de aldeído

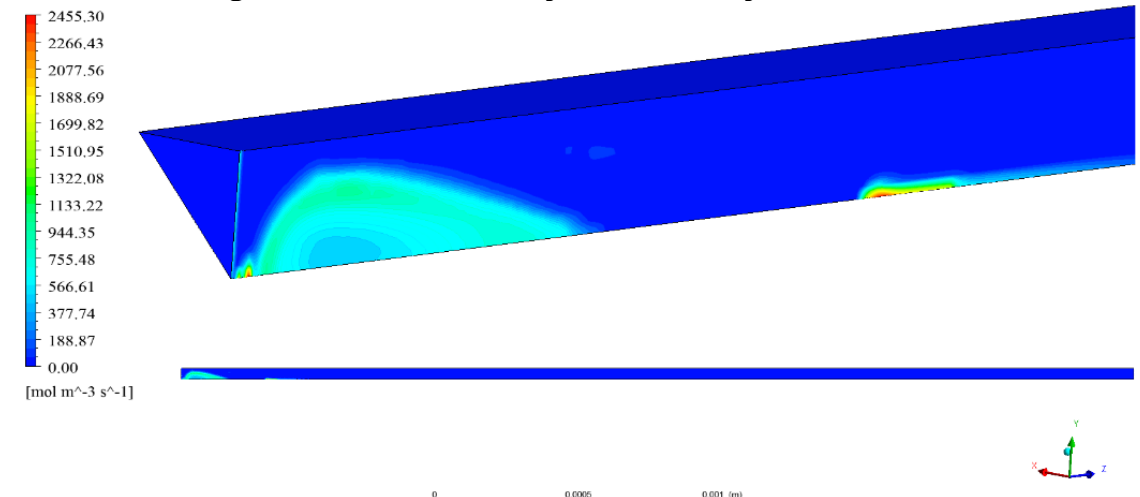

É possível observar o gradiente de temperatura ao longo do canal, característico do processo de transferência de calor associada à convecção.

Figura 4 - Perfil de temperatura no interior do microcanal

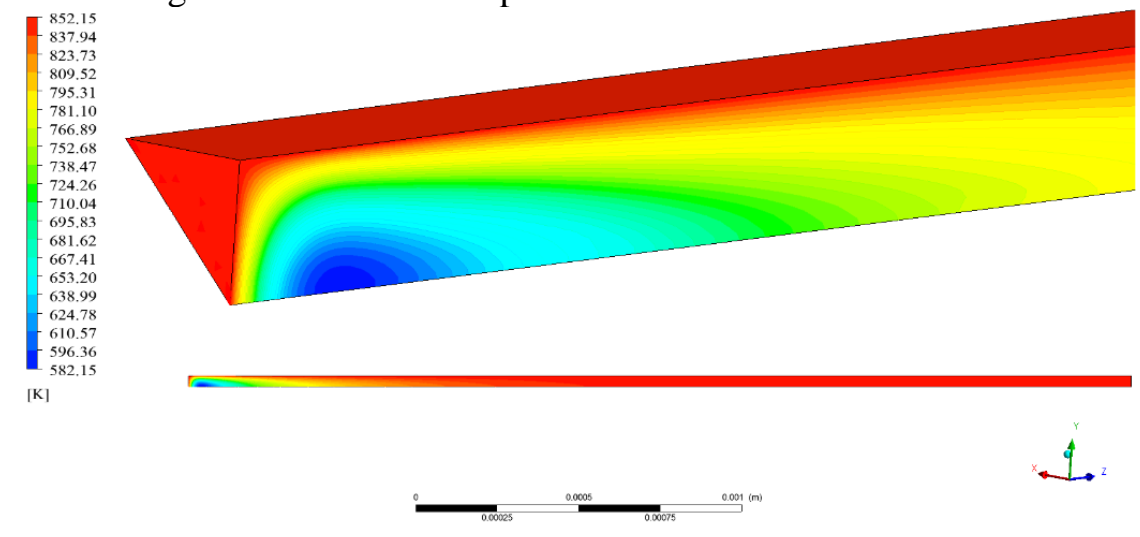

Figura 5 - Perfil de velocidade do no interior do microcanal

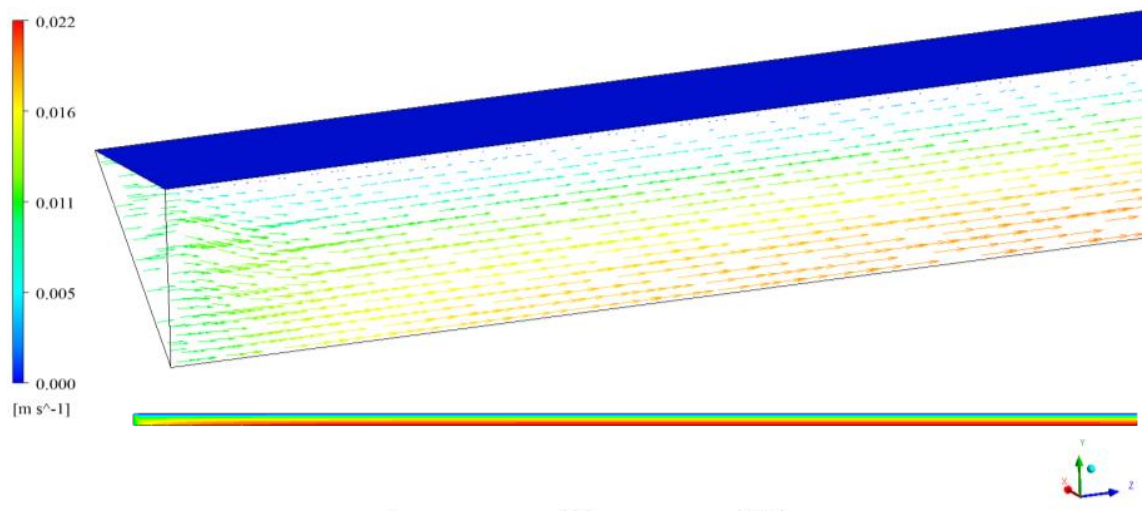

Também foram representados vetores relativos à velocidade e direção do escoamento em pontos específicos do domínio fluido, onde é possível observar que ocorre uma aglomeração das partículas na zona reativa e posterior aceleração do gás na região de saída. 


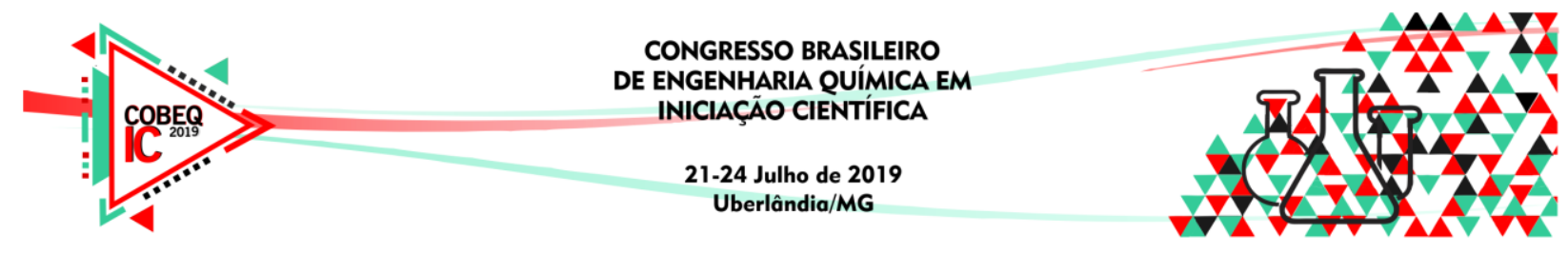

Podemos observar que o gás fica praticamente estagnado na região próxima à parede do microcanal, evidenciando a condição de não deslizamento do fluido.

\section{CONCLUSÃO}

A partir da simulação realizada, conclui-se que as condições operacionais estudadas, temperatura de $852,35 \mathrm{~K}$ e razão molar vapor/etanol de 6:1, não foram aplicáveis para o referido estudo, que visa a produção de hidrogênio. Isto porque, a reação de maior ocorrência foi a de formação do acetaldeído, atingindo taxa máxima de $2455,3 \mathrm{~mol} / \mathrm{m}^{3}$.s. A reação principal da reforma a vapor pode ter acontecido em proporções insignificantes devido ao fato destas possuírem caminhos preferenciais para ocorrer, que dependem do catalisador utilizado e das condições da reação, que, por sua vez, impactam na produção de hidrogênio. Portanto, é necessário simular diferentes condições operacionais, variando os parâmetros principais que interferem no processo, a fim de se encontrar condições em que se tem boas produções de hidrogênio e que garantam a aplicabilidade prática do reformador a vapor.

\section{REFERÊNCIAS}

ANSYS Fluent. Fluent Solver Theory Manual. Version 14.5, Ansys, 2013.

CATAPAN, R. de C. Modelagem multiescala das reações de reforma a vapor de etanol e de deslocamento água-gás sobre níquel. 119 p. Tese (Doutorado) - USP, 2009.

CHAICHAN, M. T. Performance and emission characteristics of CIE using hydrogen, biodiesel, and massive EGR. International Journal of Hydrogen Energy, Elsevier Ltd, v. 43, n. 10, p. 5415-5435, 2018.

CHEN, Z.; YAN, Y.; ELNASHAIE, S. S. E. H. Catalyst deactivation and engineering control for steam reforming of higher hydrocarbons in a novel membrane reformer. Chemical Engineering Science, v. 59, n. 10, p. 1965-1978, 2004.

MA, H. et al. Efficient hydrogen production from ethanol steam reforming over La-modified ordered mesoporous Ni-based catalysts. Applied Catalysis B: Environmental, v. 181, p. 321-331, 2016.

MAIA, T. A. et al. Produção de hidrogênio a partir da reforma a vapor de etanol utilizando catalisadores $\mathrm{Cu} / \mathrm{Ni} / \gamma$-Al2O3. Química Nova, v. 30, n. 2, p. 339-345, 2007.

MARTINS, K. C. R. Experimental, theoretical, and computational analysis of exhaust gases flow in catalytic converter platinum-palladium of internal combustion engine burning ethanol. 214 p. Tese (Doutorado) - Escola de Engenharia de São Carlos da Universidade de São Paulo, 2006.

SUN, S. et al. Thermodynamic analysis of ethanol reforming for hydrogen production. Bioenergy Systems for the Future: Prospects for Biofuels and Biohydrogen, Elsevier, v. 44, n. 1, p. 187-216, 2012.

TEIXEIRA, A. C. R. et al. Uma revisão sobre a reforma de etanol a vapor com foco na produção de hidrogênio. In: Anais do XXIV Simpósio Internacional de Engenharia Automotiva [S.1.: s.n.], 2016. p. 541-550.

VICENTE, J. et al. Reaction pathway for ethanol steam reforming on a Ni/SiO2 catalyst including coke formation. International Journal of Hydrogen Energy, v. 39, n. 33, p. 18820-18834, 2014. 